\title{
Effect of lateral heterogeneity on 2-D Rayleigh wave ZH ratio sensitivity kernels based on the adjoint method: Synthetic and inversion examples
}

\author{
Ting Lei ${ }^{1}$, HuaJian Yao ${ }^{1,2,3 *}$, and Chao Zhang ${ }^{4}$ \\ 'Laboratory of Seismology and Physics of the Earth's Interior, School of Earth and Space Sciences, University of Science and Technology of China, Hefei \\ 230026, China; \\ 2Mengcheng National Geophysical Observatory, University of Science and Technology of China, Mengcheng 233500, China; \\ ${ }^{3}$ Chinese Academy of Sciences Center for Excellence in Comparative Planetology, University of Science and Technology of China, Hefei 230026, China; \\ ${ }^{4}$ College of Oceanography, Hohai University, Nanjing 245700, China \\ Key Points: \\ - 2-D Rayleigh wave ZH ratio sensitivity kernels are calculated using the adjoint method \\ - Lateral heterogeneity has a significant impact on ZH ratio sensitivity kernels \\ - Adjoint tomography using 2-D ZH ratio kernels can resolve small anomalies as well as complex lateral structures
}

Citation: Lei, T., Yao, H. J., and Zhang, C. (2020). Effect of lateral heterogeneity on 2-D Rayleigh wave ZH ratio sensitivity kernels based on the adjoint method: Synthetic and inversion examples. Earth Planet. Phys., 4(5), 513-522. http://doi.org/10.26464/epp2020050

\begin{abstract}
The ratio between vertical and radial amplitudes of Rayleigh waves (hereafter, the Rayleigh wave ZH ratio) is an important parameter used to constrain structures beneath seismic stations. Some previous studies have explored crust and upper mantle structures by joint inversion of the Rayleigh wave ZH ratio and surface wave dispersion. However, all these studies have used a 1-D depth sensitivity kernel, and this kernel may lack precision when the structure varies a great deal laterally. Here, we present a systematic investigation of the two-dimensional (2-D) Rayleigh wave ZH ratio kernel based on the adjoint-wavefield method and perform two synthetic tests using the new kernel. The 2-D ZH ratio kernel is consistent with the traditional 1-D sensitivity kernel but has an asymmetric pattern with a preferred orientation toward the source. The predominant effect caused by heterogeneity can clearly be seen from kernels calculated from models with 2-D heterogeneities, which confirms the necessity of using the new 2-D kernel in some complex regions. Inversion tests using synthetic data show that the 2-D ZH ratio kernel has the potential to resolve small anomalies as well as complex lateral structures.
\end{abstract}

Keywords: Rayleigh wave; ZH ratio; adjoint-wavefield method; 2-D sensitivity kernel; tomography

\section{Introduction}

Rayleigh waves contain two kinds of important information, phase and amplitude information. Phase information is very stable and has been widely used in surface wave tomography (e.g., Shapiro and Ritzwoller, 2002), whereas amplitude information is more complicated and can be affected by many factors, such as the source parameters, attenuation along the ray path, and site effects (e.g., Ferreira and Woodhouse, 2007b). However, the amplitude ratios between different components are much more stable than the amplitudes themselves. The $\mathrm{ZH}$ amplitude ratio of a Rayleigh wave, which is the amplitude of the Rayleigh wave vertical component $(Z)$ over the radial component $(H)$, is one of the most commonly used amplitude ratios.

The $\mathrm{ZH}$ ratio has been used to resolve crustal structures for many

Correspondence to: H. J. Yao, hjyao@ustc.edu.cn

Received 02 NOV 2019; Accepted 24 APR 2020.

Accepted article online 24 JUL 2020.

(C)2020 by Earth and Planetary Physics. years (Boore and Toksöz, 1969), and it is a very powerful tool for investigating crustal structures (Tanimoto and Alvizuri, 2006; Tanimoto and Rivera, 2008; Yano et al., 2009), especially when combined with surface wave dispersion or even receiver functions (Lin FC et al., 2012; Chong JJ et al., 2015, 2016; Yuan Y et al., 2016; Berbellini et al., 2017; Yang $Y$ et al., 2020). The $Z H$ ratio is usually thought to be more sensitive to shallow structures compared with dispersion in the same period range (Yuan Y et al., 2016). The joint inversion from combining both dispersion and the $\mathrm{ZH}$ ratio can resolve the entire crust well. Furthermore, an increasing number of applications have used the $\mathrm{ZH}$ ratio from seismic ambient noise data in recent years (Tanimoto et al., 2013; Lin FC et al., 2014; Workman et al., 2016).

However, traditional $\mathrm{ZH}$ ratio inversion has some limitations. First, traditional approaches calculate the depth sensitivity kernel of the $\mathrm{ZH}$ ratio based on a one-dimensional (1-D) model (Tanimoto and Alvizuri, 2006; Tanimoto and Rivera, 2008; Tanimoto and Tsuboi, 2009), whereas many study regions are so complex that 1-D models are inaccurate. The bias in sensitivity kernel calculation caused 
by the complexity of these structures will then affect the inversion results. Therefore, the accuracy of the $\mathrm{ZH}$ ratio sensitivity kernel is the key to improving the accuracy and resolution of the inversion. Thus, an update from a 1-D ZH ratio kernel to the more accurate two-dimensional (2-D) or three-dimensional (3-D) ZH ratio kernel is required.

In contrast, traditional approaches consider only the fundamental mode of Rayleigh waves while ignoring the effect of overtones during calculation of the $\mathrm{ZH}$ ratio kernel (Tanimoto and Alvizuri, 2006; Tanimoto and Rivera, 2008; Tanimoto and Tsuboi, 2009). In most cases, higher modes of Rayleigh waves are believed to be much weaker than the fundamental mode, so they can be negligible, as demonstrated by Maupin (2017). But the effect of overtones is not clear and may be significant for short source-receiver distances.

Some researchers have made attempts to calculate 3-D ZH ratio sensitivity kernels. Maupin (2017) derived an expression for components perturbed by different elastic parameters and obtained the sensitivity kernels. This method gives a semi-analytical expression and has good time efficiency. However, these results are not consistent with 1-D ZH ratio sensitivity kernels. Bao XY and Shen $Y$ (2018) calculated 3-D ZH ratio sensitivity kernels by using the scattering-integral method (Zhao L et al., 2005; Chen P et al., 2007; Shen $Y$ et al., 2008; Zhang ZG and Shen Y, 2008). This method gives results compatible with those from 1-D sensitivity kernels, although its large computational cost restricts its application in inversion because of limitations in computational resources.

Two-dimensional waveform simulations using the spectral-element method show good time performance with excellent precision, even without the use of a supercomputer (Komatitsch and Tromp, 2002a, b). To add more accurate ZH ratio sensitivity kernels in a 2-D medium (instead of 1-D sensitivity kernels) to a practical inversion, we used the adjoint-wavefield method (Tromp et al., 2005; Liu QY and Tromp, 2006; Tape et al., 2007; Liu QY and Gu YJ, 2012) to calculate 2-D Rayleigh wave ZH ratio sensitivity kernels in this study. The 2-D kernels were consistent with the traditional 1-D kernels and were demonstrated to be reliable compared with the perturbation method. Moreover, we used different models to compare the $\mathrm{ZH}$ ratio sensitivity kernels to test how the $\mathrm{ZH}$ ratio sensitivity was affected by lateral structural variations. These simulations verified the necessity of replacing the 1-D sensitivity kernels with 2-D or 3-D ZH ratio sensitivity kernels in a complex medium. Two inversion scenarios using synthetic waveforms are discussed further to illustrate the performance of these new 2D sensitivity kernels.

\section{Method}

The $\mathrm{ZH}$ ratio of a Rayleigh wave is the ratio of the Z-component amplitude over the $\mathrm{H}$-component amplitude (typically at the surface) at a certain frequency $\omega$ :

$$
\eta(\omega)=\frac{A_{Z}(\omega)}{A_{H}(\omega)}
$$

Variations in the $\mathrm{ZH}$ ratio can be written as the difference between the Z-component and $\mathrm{H}$-component amplitude variations:

$$
\delta \ln \eta=\delta\left(\ln \frac{A_{Z}}{A_{H}}\right)=\delta \ln A_{Z}-\delta \ln A_{H}
$$

The measurements and kernels are related as follows (where the variation in measurements is caused only by the variation in the shear wave velocity):

$$
\begin{aligned}
& \delta \ln \eta=\int K_{\beta}^{\eta} \delta \ln \beta \mathrm{d} V, \\
& \delta \ln A_{Z}=\int K_{\beta}^{Z} \delta \ln \beta \mathrm{d} V, \\
& \delta \ln A_{H}=\int K_{\beta}^{H} \delta \ln \beta \mathrm{d} V,
\end{aligned}
$$

where $K_{\beta}^{\eta}, K_{\beta}^{Z}, K_{\beta}^{H}$ are sensitivity kernels to shear wave velocities of $\eta, A_{Z}, A_{H}$, respectively,

$$
\int K_{\beta}^{\eta} \delta \ln \beta d V=\int K_{\beta}^{Z} \delta \ln \beta \mathrm{d} V-\int K_{\beta}^{H} \delta \ln \beta \mathrm{d} V .
$$

This equation holds for any volume $V$, so the $\mathrm{ZH}$ ratio kernel can be expressed by using the Z-component and $\mathrm{H}$-component amplitude kernels:

$$
K_{\beta}^{\eta}=K_{\beta}^{Z}-K_{\beta}^{H}
$$

Similarly, we can obtain the equations for $a$ and $\rho$ :

$$
K_{a}^{\eta}=K_{a}^{Z}-K_{a}^{H}, \quad K_{\rho}^{\eta}=K_{\rho}^{Z}-K_{\rho}^{H} .
$$

Therefore, we need to calculate the sensitivity kernels of only the Z-component amplitude and $\mathrm{H}$-component amplitude, which can be solved by using the adjoint-wavefield method (Tromp et al., 2005; Liu QY and Tromp, 2006; Liu QY and Gu YJ, 2012). The amplitude adjoint source is given by

$$
s^{\dagger}(T-t, \omega)=\frac{1}{M} G[w(t) s(t), \omega]
$$

where $w(t)$ is the cross-correlation time window, $s(t)$ is the waveform recorded by a station, $G[\cdot, \omega]$ denotes Gaussian filtering at the central frequency $\omega$, and $M$ is the normalization factor:

$$
M=\int_{0}^{T} G[w(t) s(t), \omega]^{2} \mathrm{~d} t .
$$

According to the expression of the adjoint source, everything in the selected time window $w(t)$ will contribute to the final sensitivity kernel. In practice, other types of waves, such as $S$ waves and higher mode Rayleigh waves, are not always muted by the window, especially for short source-receiver distances, so this kernel is different from the traditional one, which includes only the contribution of fundamental-mode Rayleigh waves.

The definition of amplitude ratio in this paper is noteworthy. The maximum amplitude of the envelope has commonly been used in ZH ratio studies (Lin FC et al., 2014; Yuan Y et al., 2016; Yang Y et al., 2020):

$$
\eta(\omega)=\frac{A_{z}^{\prime}(\omega)}{A_{H}^{\prime}(\omega)}=\frac{\max \left(E\left[u_{z}(\omega)\right]\right)}{\max \left(E\left[u_{H}(\omega)\right]\right)^{\prime}}
$$

where $u$ denotes the filtered waveform, with $u=G[w(t) s(t), \omega]$, and $E[u]$ denotes the envelope of $u$ (Figure 1). In this study, we used the following definition (Dahlen and Baig, 2002; Ferreira and Woodhouse, 2007a): 

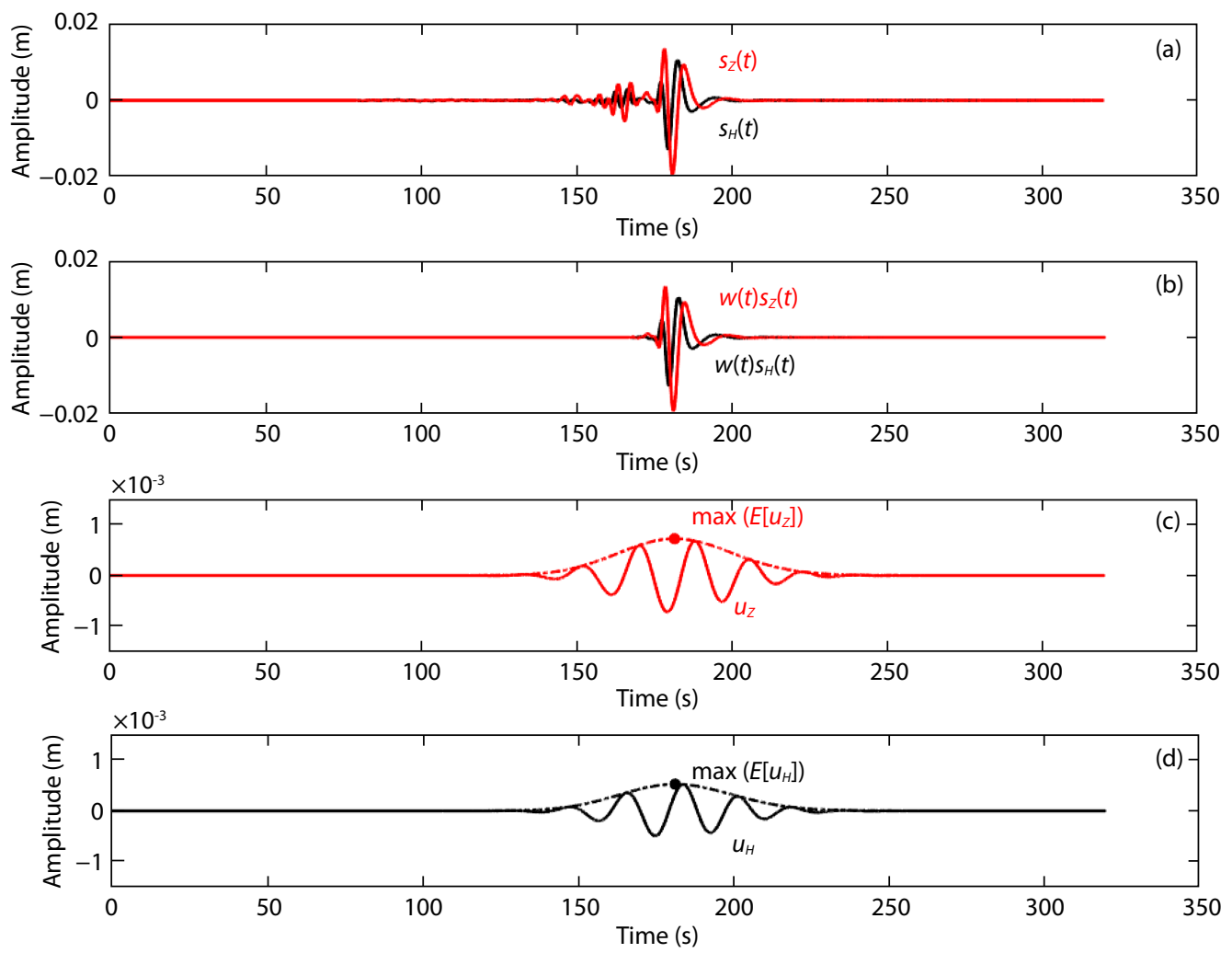

Figure 1. Procedure for obtaining the adjoint source. (a) Waveforms recorded by a station; the red trace represents the Z-component, and the black trace represents the H-component. (b) Waveforms with body waves muted with the window function $w(t)$. (c) Z-component waveform filtered by a Gaussian filter (solid line) and its waveform envelope (dashed line). (d) H-component waveform filtered by a Gaussian filter (solid line) and its waveform envelope (dashed line).

$$
\eta(\omega)=\frac{A_{Z}(\omega)}{A_{H}(\omega)}=\sqrt{\frac{\int_{0}^{T} u_{Z}^{2}(\omega) \mathrm{d} t}{\int_{0}^{T} u_{H}^{2}(\omega) \mathrm{d} t} .}
$$

Both definitions are valid and give similar $\mathrm{ZH}$ ratio values, and we need only guarantee that we are using the same definition in kernel calculation and data processing. From Equations (11) and (12), the $\mathrm{ZH}$ ratio measurements are obviously frequency dependent. Existing methods, such as the multi-taper method (Zhou $Y$ et al., 2004), can be used to make such frequency-dependent measurements.

\section{Results}

\subsection{2-D ZH Ratio Sensitivity Kernel and Its Validation}

\subsubsection{Sensitivity kernels of the $\mathrm{ZH}$ ratio}

By subtracting the $\mathrm{H}$-component amplitude sensitivity kernel from the Z-component amplitude sensitivity kernel (Equation (7)), we can obtain the sensitivity kernel of the $\mathrm{ZH}$ ratio (Figure 2 ). We use a constant model in the simulation. The shear wave velocity is $3,200 \mathrm{~m} / \mathrm{s}$. The compressive wave velocity and density are determined by empirical formulas (Brocher, 2005).

On the source side, the Z-component and H-component amplitudes have the same sensitivity pattern, so they cancel each other out in the final $\mathrm{ZH}$ ratio sensitivity kernel. The $\mathrm{ZH}$ ratio sensitivity concentrates on the station side, which is in accordance with previous studies, that is, the $\mathrm{ZH}$ ratio is sensitive to the structure beneath the station. However, the $\mathrm{ZH}$ ratio sensitivity kernel has a shape oriented toward the source.

The $\mathrm{ZH}$ ratio sensitivities to different model parameters at different periods are also calculated as shown in Figure 3. It is obvious that $\mathrm{ZH}$ ratios have little sensitivity to compressional wave velocity (Figure 3a), whereas they are sensitive to both shear wave velocity and density. Density is closely related to geologic structure. Although it is not easy to constrain the density from seismic tomography, the $\mathrm{ZH}$ ratio can provide some helpful information on a shallow density structure (Lin FC et al., 2012, 2014).

Two-dimensional $\mathrm{ZH}$ ratio sensitivity kernels provide more information than 1-D sensitivity kernels, and the most important one is the lateral resolution. One-dimensional $\mathrm{ZH}$ ratio sensitivity kernels do not consider the source direction and do not have lateral variation, but 2-D ZH ratio kernels are laterally varied and can resolve complex lateral structures, which is very important in some complicated regions.

In a traditional inversion using $\mathrm{ZH}$ ratio measurements, we need to extract the $\mathrm{ZH}$ ratios of one station by using waveforms from many different sources from different azimuths. We then average them to obtain the average and azimuthally independent $\mathrm{ZH}$ ratios. From the 2-D ZH ratio kernels, we can see the reason for this. The 1-D sensitivity kernel can be seen as the average kernel from different azimuths, whereas the $\mathrm{ZH}$ ratio sensitivity kernel from the single source-station pair has a certain preferred azimuth (toward the source). Therefore, the $\mathrm{ZH}$ ratio values should be aver- 


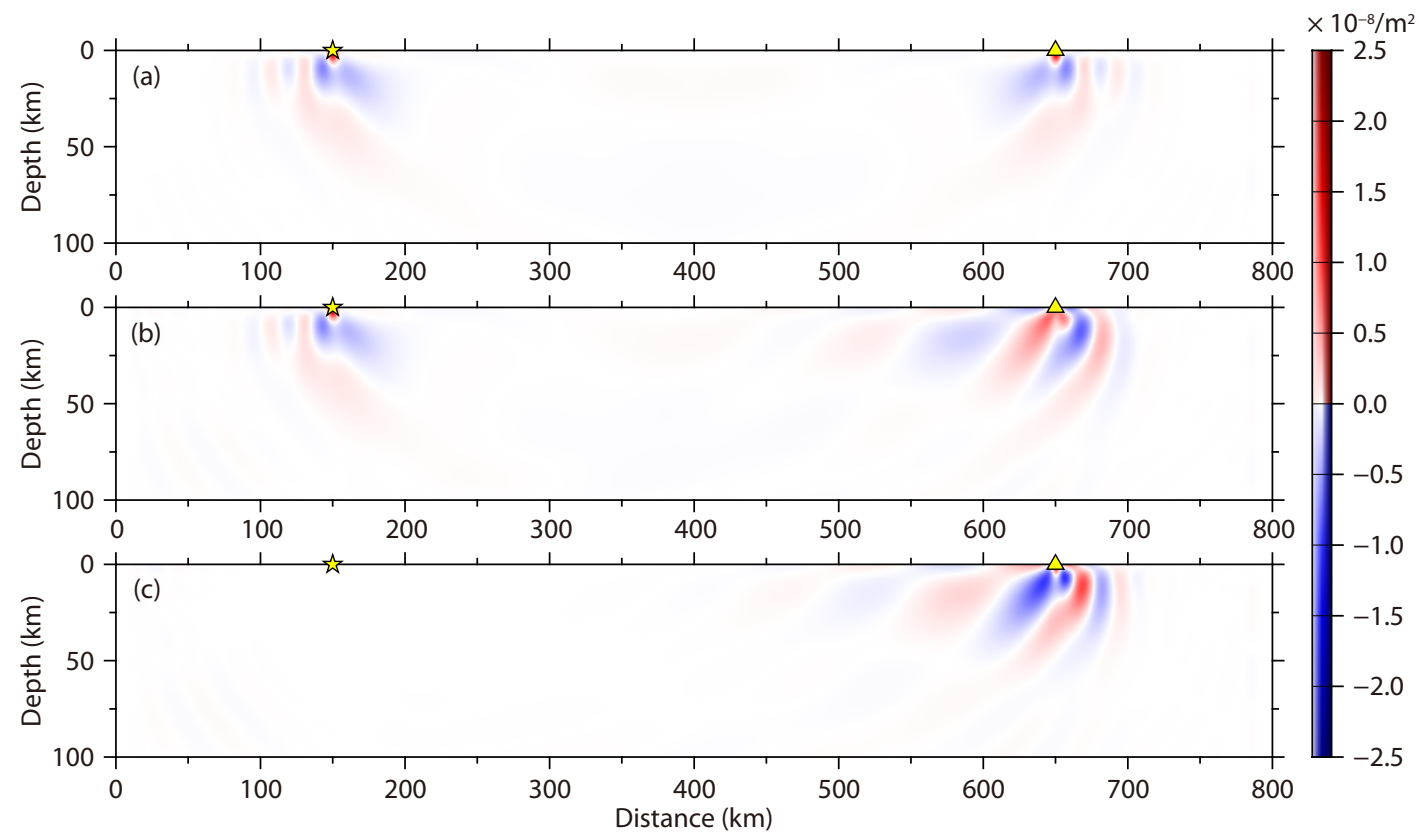

Figure 2. Illustration of 2-D ZH ratio sensitivity kernel computation to shear wave velocity for Rayleigh waves (at the central period of $20 \mathrm{~s}$ ). (a) Zcomponent amplitude sensitivity. (b) H-component amplitude sensitivity. (c) ZH ratio sensitivity. The source is shown as a star and the receiver is shown as a triangle.

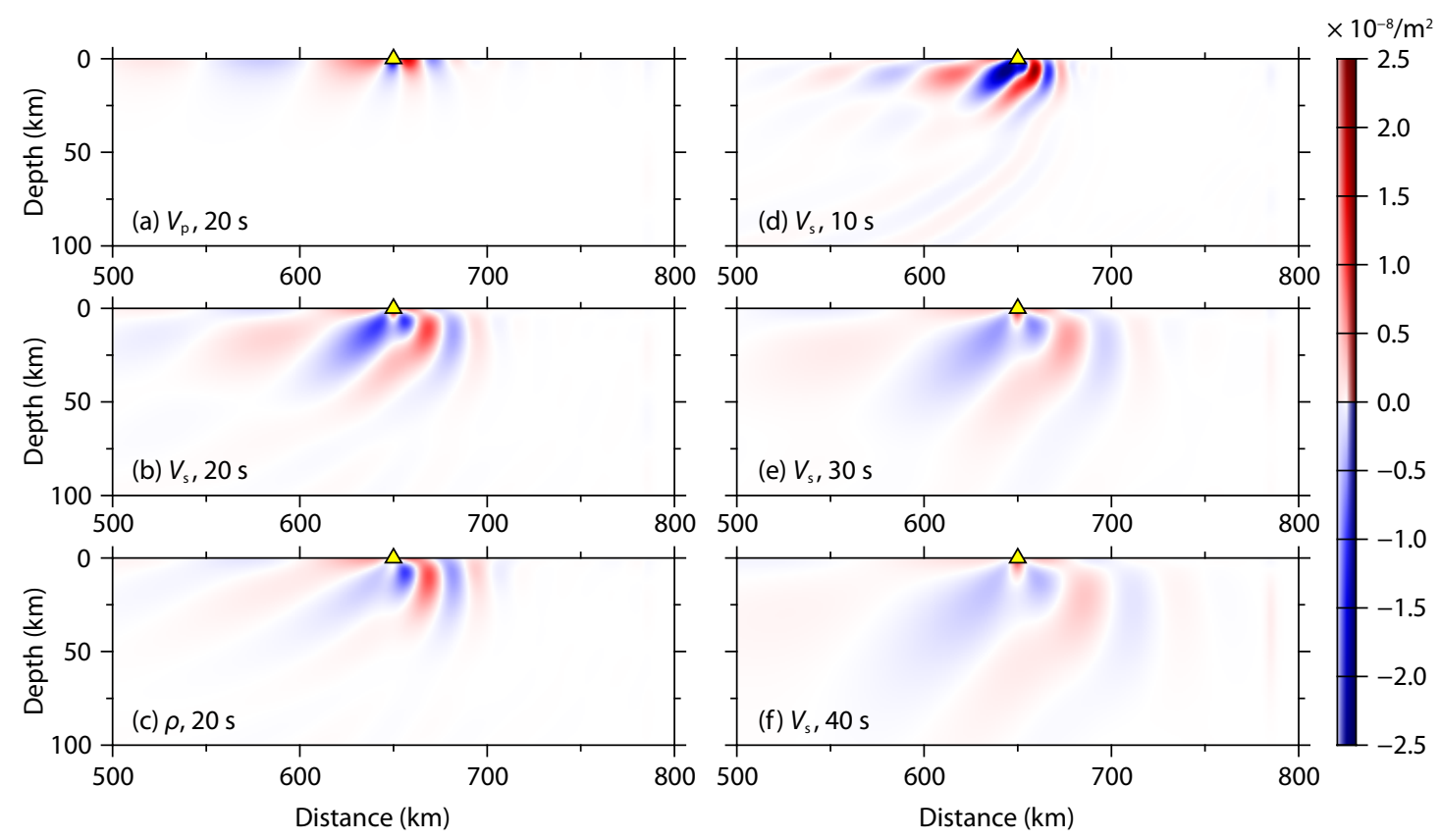

Figure 3. The ZH ratio sensitivity kernels to different model parameters at different periods. Sensitivity to (a) compressional wave velocity, (b) shear wave velocity, and (c) density at the period of $20 \mathrm{~s}$. (d-f) ZH ratio sensitivity to shear wave velocity at different periods: $10 \mathrm{~s}$ (d), $30 \mathrm{~s}$ (e), and $40 \mathrm{~s}$ (f).

aged to match the 1-D sensitivity kernels. The new 2-D kernels also demonstrate the importance of the source azimuth distribution in traditional $\mathrm{ZH}$ ratio measurements. If the sources are on one side of the station, the $\mathrm{ZH}$ ratio value will reflect the structure of that particular side of the station, rather than the average structure beneath the station. This incompatibility between the data and kernel may cause some problems in the inversion.
We can now bypass this issue, and the new 2-D kernel can make full use of the $\mathrm{ZH}$ ratio values for every source-station pair. However, the $\mathrm{ZH}$ ratio from a single source-station pair is not as stable as the azimuthally averaged value.

3.1.2 Validation of the $\mathrm{ZH}$ ratio kernel

The perturbation method is a basic method used to evaluate sens- 
itivity kernels (Tanimoto and Alvizuri, 2006; Tanimoto and Rivera, 2008; Tanimoto and Tsuboi, 2009). We used the adjoint-wavefield method to calculate the sensitivity kernel, and we used the perturbation method to validate the results.

We added perturbations at different locations in the model, as shown in Figure 4a. Line 1 is a lateral profile and Line 2 is a vertical profile. The original shear velocity is $3200 \mathrm{~m} / \mathrm{s}$, and the perturbed velocity is $3400 \mathrm{~m} / \mathrm{s}$ for each $20 \mathrm{~km} \times 20 \mathrm{~km}$ square region in the 2 D model.

On the one hand, we can perform the simulation by using the original model and the perturbed model to obtain the waveforms. We can then measure the $\mathrm{ZH}$ ratio and calculate the $\mathrm{ZH}$ ratio perturbation caused by the shear wave velocity perturbation. On the other hand, because we have already calculated the 2-D sensitivity kernel from the adjoint method, we can predict the $\mathrm{ZH}$ ratio perturbation by using the formula below:

$$
\delta \ln \eta=\int K_{\beta}^{\eta} \delta \ln \beta \mathrm{d} S,
$$

where the surface integral covers the perturbed region. Therefore, we can compare the $\mathrm{ZH}$ ratio perturbations obtained by these two approaches, as shown in Figure $4 \mathrm{~b}$ and $4 \mathrm{c}$.

As shown in Figure 4b, the two results (red line and blue circles) were somewhat different. This may be because (1) our kernel was based on the first-order approximation, and the velocity perturbations we added were not infinitesimally small, which may have introduced some biases; and (2) some numerical calculation errors (including model parameterization, the surface integral, and so on) may have played a minor part in the total error.

In general, the difference was less than $2 \%$, so the simulation results were in accordance with the predicted results. The sensitivity kernel based on the adjoint method was accurate enough to be used in the inversion.

The scattering-integral method (Zhao $L$ et al., 2005; Chen $P$ et al., 2007; Shen Y et al., 2008; Zhang ZG and Shen Y, 2008) and the adjoint-wavefield method (Tromp et al., 2005; Liu QY and Tromp, 2006; Liu QY and Gu YJ, 2012) are both efficient in sensitivity kernel calculation. In this case, both methods gave reliable $\mathrm{ZH}$ ratio kernels (Bao XY and Shen Y, 2018). The choice between these two methods should be made according to the number of sources and receivers (Chen $P$ et al., 2007).

The most popular 1-D ZH ratio sensitivity kernels are calculated based on the perturbation method for the 1-D model, so we compared our 2-D kernels with the 1-D kernels (Figure 5). The 2-D sensitivity kernels were laterally summed to compare them with
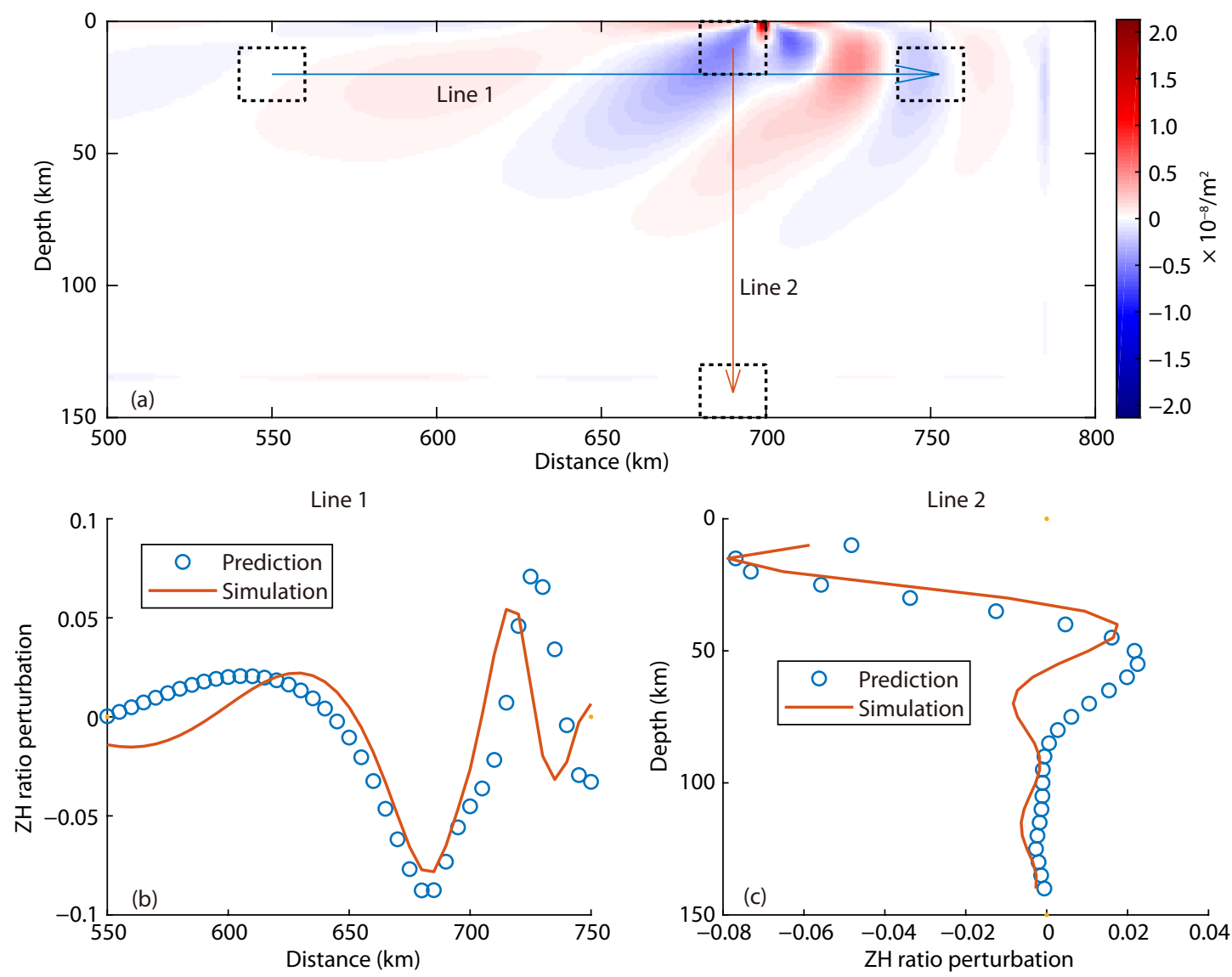

Figure 4. Comparison of $\mathrm{ZH}$ ratio perturbation by using two different methods. (a) The location of two profiles (lateral profile, Line 1, and vertical profile, Line 2) where we added a velocity perturbation (the dashed box). (b) ZH ratio perturbation comparison for the lateral profile, Line 1. The red line denotes the $\mathrm{ZH}$ ratio perturbation measured from the forward simulation waveforms, whereas the blue circles denote the $\mathrm{ZH}$ ratio perturbation predicted from the definition of the sensitivity kernel (Equation (13)). (c) Same as (b) but for the vertical profile, Line 2. 
the 1-D kernels, that is, the summed kernel values at the same depth:

$$
K_{\beta}^{\eta_{110}}(z)=\int K_{\beta}^{\eta}(x, z) d x
$$

Hence,

$$
\delta \ln \eta=\int K_{\beta}^{\eta_{1 \mathrm{D}}}(z) \delta \ln \beta(z) \mathrm{d} z
$$

The laterally summed results were consistent with the 1-D results (Figure 5). In fact, the higher modes of Rayleigh waves could not be removed in the simulation process, whereas the calculation of the 1-D kernel considered only the fundamental mode. The results showed that the higher modes did not contribute much to the $\mathrm{ZH}$ ratio sensitivity in our case with a source-receiver distance of $600 \mathrm{~km}$.

The 2-D ZH ratio sensitivity kernels had a similar sensitivity depth as Rayleigh wave dispersion in the same period, which was different from the traditional 1-D ZH ratio kernels (e.g., Yuan $\mathrm{Y}$ et al., 2016). The 1-D ZH ratio kernel at $20 \mathrm{~s}$ showed no sensitivity at around $30 \mathrm{~km}$ (Figure 5), but the 2-D kernel (also at $20 \mathrm{~s}$ ) still showed some sensitivity at $50 \mathrm{~km}$. Because the 1-D kernel was laterally summed from the 2-D kernel, the sensitivity of the deeper part of the 2-D kernel would be canceled when laterally summed.

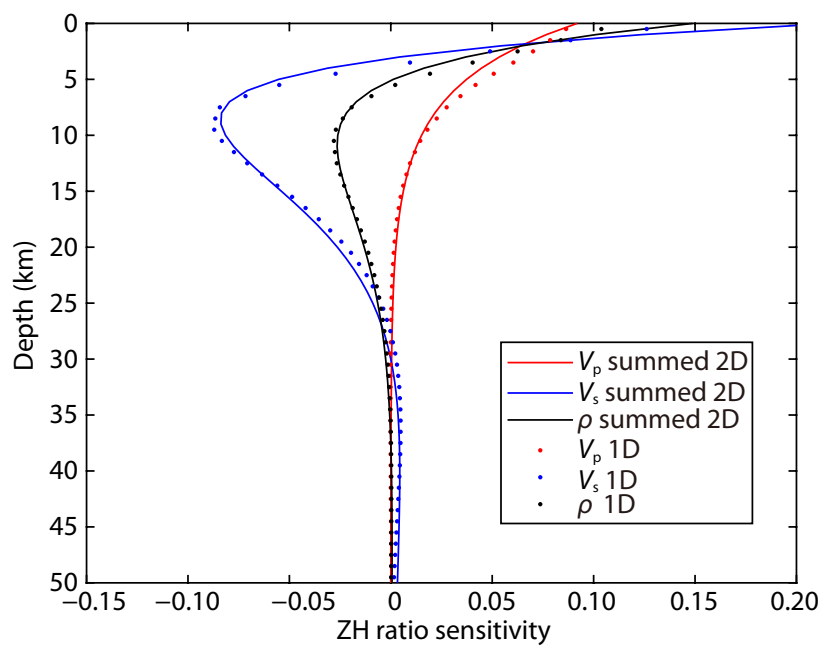

Figure 5. Comparison of the laterally summed 2-D ZH ratio sensitivity kernels (solid lines) and 1-D kernels (dotted lines) in a $20 \mathrm{~s}$ period for different parameters.

\subsection{Effect of Lateral Heterogeneity on 2-D ZH Ratio Kernels}

The 1-D sensitivity kernels of the $\mathrm{ZH}$ ratio are easy to calculate, whereas the 2-D kernels take much more computational time but are still sustainable. The 2-D sensitivity kernels of the $\mathrm{ZH}$ ratio should be calculated for the reason that although the 1-D model is a good approximation in most cases, many regions of the structures beneath are far away from simple 1-D layered structures, such as the vicinity of faults and the boundary area between tectonic blocks. If we still use the sensitivity kernels based on 1-D models, the kernels may not be accurate enough.
We chose two typical structures, one with an undulated interface and the other with a velocity anomaly, to demonstrate such an effect. The results are shown in Figures 6 and 7.

The undulated interface can obviously affect the $\mathrm{ZH}$ ratio sensitivity (Figure 6). The sensitivity above the interface is obviously higher than the sensitivity beneath the interface (Figure 6). In this case, the region most affected is region $A$ in Figure 6, where the sensitivity intensity is about $50 \%$ greater than that of the flat interface model at the same location.

The effect of a velocity anomaly is very complex (Figure 7). We added a high-velocity anomaly $(3,400 \mathrm{~m} / \mathrm{s}, 20 \mathrm{~km} \times 20 \mathrm{~km}$ square) to the original model with a constant velocity of $3,200 \mathrm{~m} / \mathrm{s}$. The sensitivity difference between the two models is mainly focused on the anomaly, and there is a little influence around it. The sensitivity difference is about $20 \%$.

In summary, both undulated interfaces and velocity anomalies can affect the sensitivity kernel, whereas the accuracy of sensitivity kernels is the core of the inversion and will affect the inversion result. In practice, velocity structures are very complex, particularly for the crust. Therefore, using a more accurate kernel is an important approach to improving the accuracy of the inversion.

In the previous examples, the source we used for the waveform simulation was an acoustic source. Here, we also performed a series of tests to clarify the effect of the source parameter. In the test, both the source and receiver were located on the surface. We defined the angle between the nodal plane and the horizontal plane as $\theta$. When $\theta>1^{\circ}$, the sensitivity kernels were very similar, and the difference was less than $1 \%$. But when $\theta=0^{\circ}$ (the receiver was exactly in the direction of the nodal plane), the sensitivity ker-
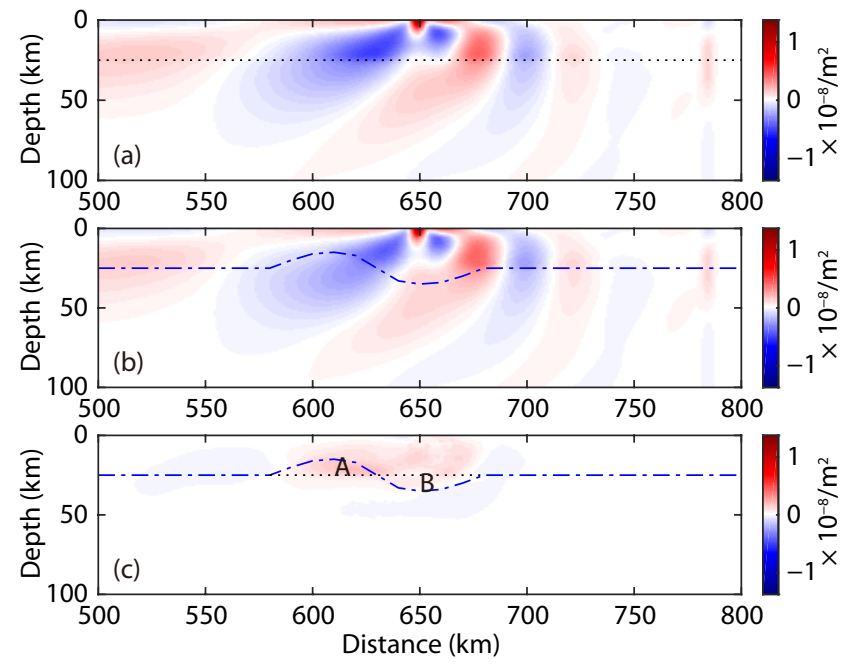

Figure 6. Effect of an undulated interface on the $\mathrm{ZH}$ ratio kernel. (a) The $\mathrm{ZH}$ ratio kernel simulated by using a flat interface model. (b) The $\mathrm{ZH}$ ratio kernel simulated by using an undulated interface model. (c) Difference between (a) and (b). The flat and undulated interfaces are shown as dotted and dashed-dotted lines, respectively. The source is located at $(150 \mathrm{~km}, 0 \mathrm{~km})$ and the receiver is at $(650 \mathrm{~km}, 0 \mathrm{~km})$. The shear wave velocity is $3,200 \mathrm{~m} / \mathrm{s}$ above the interface and $4,200 \mathrm{~m} / \mathrm{s}$ below the interface. 

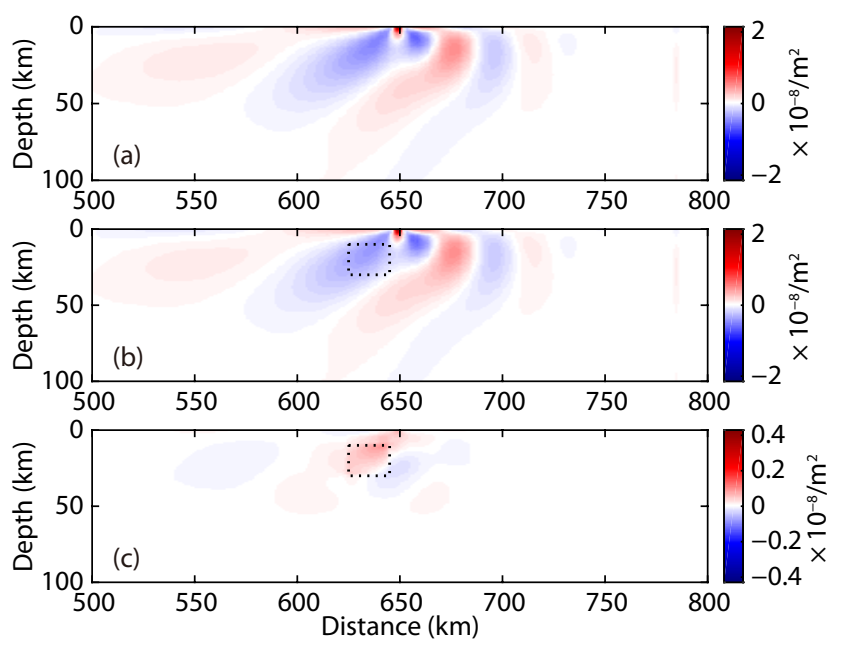

Figure 7. Effect of a velocity anomaly on the $\mathrm{ZH}$ ratio sensitivity kernel. (a) The ZH ratio kernel simulated by using a flat model with a constant shear wave velocity of 3,200 m/s. (b) The ZH ratio kernel simulated by using a model with a velocity anomaly $(3,400 \mathrm{~m} / \mathrm{s})$, as shown by the dashed-line box. (c) Difference in kernels between (a) and (b).

nel was singular and the $\mathrm{ZH}$ ratio measurement was not reliable. This test may motivate us when processing $\mathrm{ZH}$ ratio measurements. The radiation pattern will affect the $\mathrm{ZH}$ ratio measurement significantly only when the receiver is in nearly the same direction as the nodal plane. Thus, we need to avoid this case when making $\mathrm{ZH}$ ratio measurements.

\subsection{2-D Inversion Using the ZH Ratio from Synthetic Data}

The $\mathrm{ZH}$ ratio is an important and independent measurement used to constrain velocity structures. Here, we used synthetic data to demonstrate the successful inversion of 2-D shear wave velocity models by using adjoint-based 2-D ZH ratio sensitivity kernels.

\subsubsection{Inversion using seismic profile data}

A linear seismic profile is the most suitable situation for applying a 2-D inversion using the 2-D ZH ratio kernels. Seismic profile data have been increasing in recent years. Although it is difficult to find enough earthquakes along the profile, researchers have developed an approach in which the $\mathrm{ZH}$ ratio is extracted from noise cross-correlation data (Lin FC et al., 2014; Workman et al., 2016). The reconstructed empirical Green's function can provide some information on the $\mathrm{ZH}$ ratio, although the instability of $\mathrm{ZH}$ ratios from single virtual source-receiver pairs is an issue compared with dispersion measurements.

We set up a 2-D profile that was $800 \mathrm{~km}$ in length and $150 \mathrm{~km}$ in depth. Receivers were set from 150 to $650 \mathrm{~km}$, with $10 \mathrm{~km} \mathrm{spa-}$ cing. Any station could be considered a virtual source, and the $\mathrm{ZH}$ ratios of other stations could be measured. To separate Rayleigh waves from body waves, we used only data satisfying $d>2.5 \lambda$, where $d$ is the distance of the station pair and $\lambda$ is the wavelength of the period we used. We used only the $\mathrm{ZH}$ ratio at four central periods $(10,20,30$, and $40 \mathrm{~s})$ to demonstrate the process of inversion. In fact, these four periods were able to cover the period band of 6 to 45 s quite well because of the use of a Gaussian filter.
We chose the linear least squares inversion method, which requires more computational time. For example, we needed to calculate $2 N_{\text {event }} \times N_{\text {receiver }} \times N_{\text {period }}$ kernels to use this approach, whereas we needed to calculate only $N_{\text {event }}+3 N_{\text {receiver }}$ simulations to use adjoint inversion (Tape et al., 2007). However, adjoint inversion can fall into local minima more easily. The $\mathrm{ZH}$ ratio had very large sensitivity around the station, which aggravated this situation. Generally, we needed to smooth the kernel to reduce the possibility that it would fall into local minima. This was difficult for the $\mathrm{ZH}$ ratio kernels. If we used little smoothing, it would be insufficient to prevent the kernel from being trapped in local minima. If we used strong smoothing, the smoothed $\mathrm{ZH}$ ratio kernel might be totally different from the original one because the 2D kernel had oscillating positive and negative values. However, adjoint inversion using the new kernels was still a possible direction for improving the computational performance time if we could find another way to prevent the inversion from falling into local minima, such as by using a good starting model, as many previous studies have done.

In our synthetic data example, we set up three anomolies that were $40 \mathrm{~km}$ in length and $20 \mathrm{~km}$ in width at around $20 \mathrm{~km}$ deep. The background velocity was $3,200 \mathrm{~m} / \mathrm{s}$, the high velocity was $3,400 \mathrm{~m} / \mathrm{s}$, and the low velocity was $3,000 \mathrm{~m} / \mathrm{s}$. The initial model was a simple constant model with a velocity of $3,200 \mathrm{~km} / \mathrm{s}$.

The model was discretized to $M$ model parameters, and the inversion model grids were $5 \mathrm{~km} \times 5 \mathrm{~km}$ (represented by $\Omega_{1}, \Omega_{2}, \ldots \Omega_{M}$ ). The $G$ matrix was constructed by

$$
\boldsymbol{G}_{i j}=\int \boldsymbol{K}_{i} \boldsymbol{B}_{j} \mathrm{~d} S,
$$

where $\boldsymbol{B}_{j}$ denotes the basis function, and $\boldsymbol{j}$ is an index of the discretized model parameter $(1 \leq j \leq M)$ :

$$
\boldsymbol{B}_{j}= \begin{cases}1, & \text { when } \mathrm{d} S \in \Omega_{j}, \\ 0, & \text { when } \mathrm{d} S \notin \Omega_{j} .\end{cases}
$$

The inversion was carried out with the zero-order Tikhonov regularization, and the variation of the model in the inversion was

$$
\delta \boldsymbol{m}=\left(\boldsymbol{G}^{\top} \boldsymbol{G}+a^{2} \boldsymbol{I}\right)^{-1} \boldsymbol{G}^{\top} \delta \boldsymbol{d} .
$$

First, we did the inversion with only $\mathrm{ZH}$ ratio data (Figure $8 \mathrm{~b}$ ). The anomolies were generally reconstructed, but they were not sufficient. Some smearing effects occurred, and the model exhibited some artifacts at deeper depths, whereas the shallow depth structure recovered well. This gave us confidence in using the new $\mathrm{ZH}$ ratio kernel for structural inversion.

We knew that phase information on surface waves could also be used to invert for velocity structures. Zhang C et al. (2018) applied an adjoint inversion to the ambient noise cross-correlation traveltime data of a seismic profile and obtained a 2-D velocity model beneath the profile. We first performed a test to compare the recovered model using $\mathrm{ZH}$ ratios and the model using cross-correlation travel times. The recovered model using travel-time data was much smoother.

To constrain both the shallow and deeper structures, we performed a joint inversion using both cross-correlation travel-time and $\mathrm{ZH}$ ratio data (Figure $8 \mathrm{~d}$ ). The contributions of the travel-time 

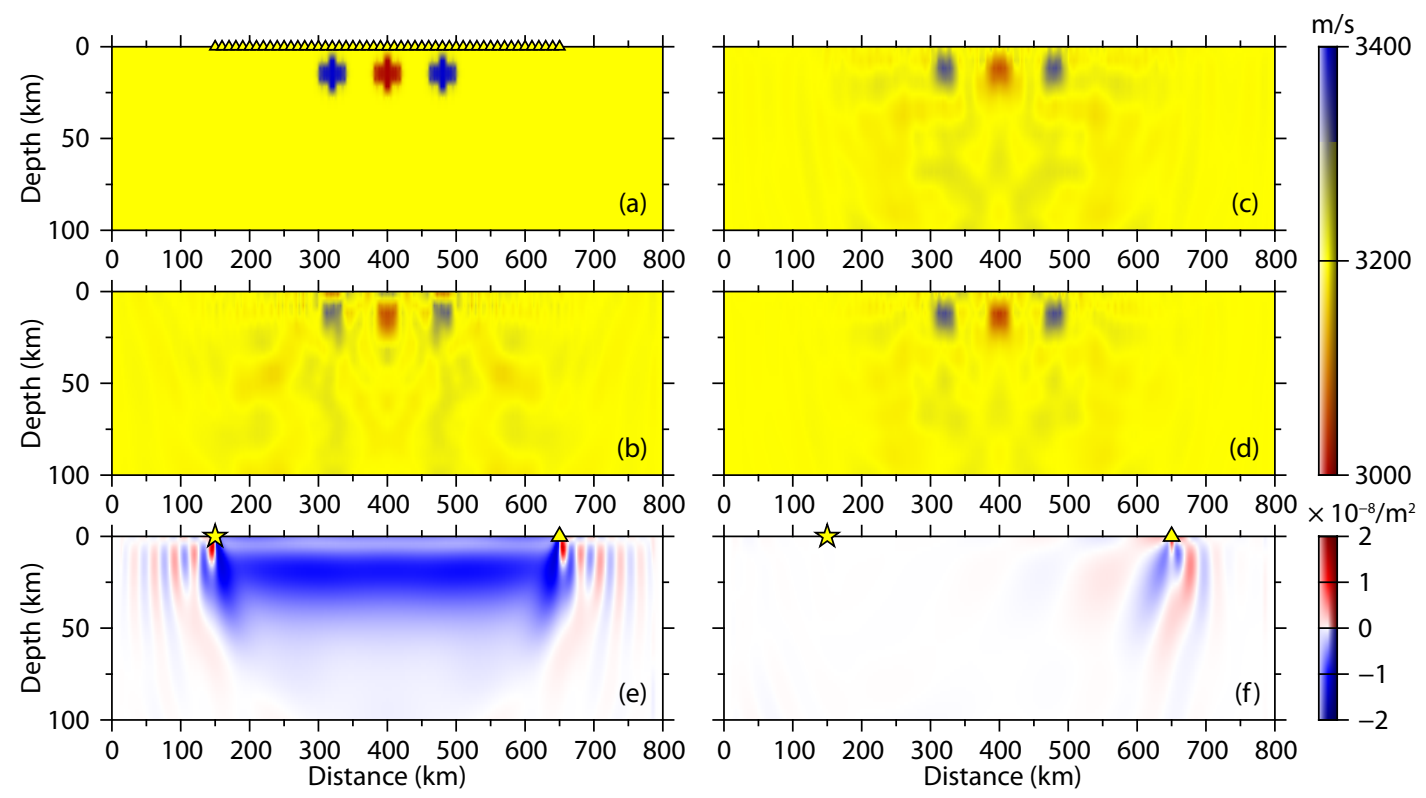

Figure 8. Demonstration of inversion by using the ZH ratio data and 2-D kernels. (a) The target model, with three velocity anomalies at around $20 \mathrm{~km}$ depth. The triangles are stations. (b) The inversion result using only ZH ratio data. (c) The inversion result using only the cross-correlation travel-time data of Rayleigh waves. (d) The joint inversion result using both the ZH ratio and cross-correlation travel-time data. (e) Example of the Rayleigh wave travel-time sensitivity kernel in the period band of 19 to $31 \mathrm{~s}$. The star denotes the virtual source and the triangle is the receiver. (f) Example of the $\mathrm{ZH}$ ratio kernel at the central period of $30 \mathrm{~s}$.

data $\left(\delta \boldsymbol{d}_{\mathrm{TM}}\right)$ and $\mathrm{ZH}$ ratio data $\left(\delta \boldsymbol{d}_{\mathrm{ZH}}\right)$ were balanced by a weighting factor, $\xi$ :

$$
\left(\begin{array}{c}
\xi \boldsymbol{G}_{\mathrm{TM}} \\
(1-\xi) \boldsymbol{G}_{\mathrm{ZH}}
\end{array}\right) \delta m=\left(\begin{array}{c}
\xi \delta \boldsymbol{d}_{\mathrm{TM}} \\
(1-\xi) \delta \boldsymbol{d}_{\mathrm{ZH}}
\end{array}\right) .
$$

In this study, we used $\xi=0.5$. The recovered model (Figure $8 \mathrm{~d}$ ) was much better than the two prior results (Figure 8b, 8c). The upper surface and lower surface were both well resolved. With two different kinds of data, the model was better constrained, with much less smearing.

In actuality, seismic waves propogate through 3-D media. If we want to use the data in a 2-D framework, we need to do a waveform transformation from 3-D to 2-D (Forbriger et al., 2014). For the phase part, the correction (known as a $\pi / 4$ phase shift) was previously tested by Zhang $C$ et al. (2018). For the amplitude part, the correction is related to the distance between the source and receiver and is the same for the $\mathrm{Z}$-component and the H-component. Thus, no amplitude correction is needed in the 2-D framework.

\subsubsection{Inversion test using teleseismic/regional earthquake data} The kernel of the $\mathrm{ZH}$ ratio is very similar to the travel-time difference kernel (Yuan YO et al., 2016; Bao XY and Shen Y, 2018). If we want to investigate the local structure but lack local events, the teleseismic/regional earthquake travel-time difference and $\mathrm{ZH}$ ratio data are very useful in this situation.

Below, we display one synthetic test using the $\mathrm{ZH}$ ratio of a regional earthquake Rayleigh wave. There are two sources, one at each side of the study region (Figure 9a). The study region is strongly heterogeneous, so the 1-D assumption is not appropri- ate. But with the 2-D ZH ratio kernel, we can easily recover the checkerboard anomalies. The lateral resolution is also very good (Figure 9b).

We have mentioned that the $\mathrm{ZH}$ ratio measurements usually have large uncertainties. Thus, we tested the performance of the inversion when adding 5\% measurement error, and the result was also acceptable (Figure 9c). The error of a single source-station pair $\mathrm{ZH}$ ratio measurement was sometimes greater than $10 \%$. If we could reduce the measurement error of the $\mathrm{ZH}$ ratio to several percentage points, this approach would be very useful in some real applications.

\section{Discussion}

Calculation of the 2-D ZH ratio kernels was relatively efficient. For the kernel simulation, if the model was $800 \mathrm{~km}$ in length and 150 $\mathrm{km}$ in depth with $160 \times 50$ elements, the simulation contained 12,000 time steps with a $0.02 \mathrm{~s}$ time interval, and the computational time cost was about 2 central processing unit (CPU) hours (AMD Opteron 6376). But if you wanted to reach the same precision in a 3-D case (the model was $800 \mathrm{~km}$ in length, $50 \mathrm{~km}$ in width, and $150 \mathrm{~km}$ in depth, with $160 \times 10 \times 50$ elements), it would take about 3,750 CPU hours. On the basis of the time cost, the 2-D kernels are very efficient when computational resources are limited.

The synthetic examples above provide us with several actual situations for the use of $\mathrm{ZH}$ ratio kernels. We can use ambient noise data from linear seismic profiles with no limitation on the distribution of seismic events. However, the effectiveness of this method in real practice still requires more study because of the difficulty of extracting stable $\mathrm{ZH}$ ratio measurements from a single source-sta- 


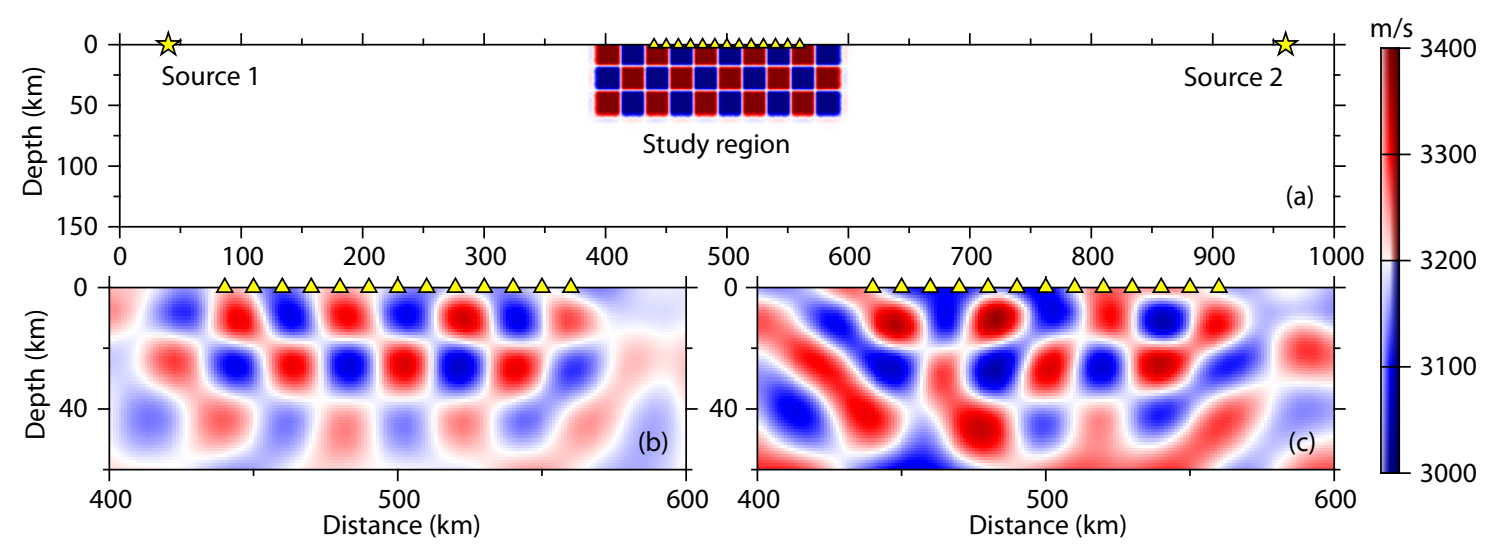

Figure 9. ZH ratio adjoint tomography using regional events outside the study region. (a) The observation system and study region. The two sources are shown as stars, and the stations are denoted by triangles. (b) The recovered model using noiseless ZH ratio data. (c) The recovered model using $\mathrm{ZH}$ ratio data with $5 \%$ random error.

tion pair. Similarly, using amplitude information from an ambient noise cross-correlation is not always reliable (Allmark et al., 2018).

In addition, if we used active sources, this method could be used effectively because we need to measure only amplitude ratios, which are not limited by the source time function. We could use not only the phase information, as is commonly done in a fullwaveform inversion, but also the amplitude ratio information, as shown in this study. The biggest problem with using the 2-D ZH ratio kernels is the reliablity of the $\mathrm{ZH}$ ratio measurements from a single source-station pair. However, some active source experiments are repeatable, which makes the $\mathrm{ZH}$ ratio measurement more stable.

Another issue is the 3-D structural effect on the $\mathrm{ZH}$ ratio kernels, which are not properly modeled in the 2-D case. For example, the ray path may not follow the profile. Thus, the requirements for the linear profile need to be considered before a field experient is conducted.

Joint inversion with both surface wave dispersion and $\mathrm{ZH}$ ratio data is very effective (Lin FC et al., 2012; Chong JJ et al., 2015; Yuan $Y$ et al., 2016). The $Z H$ ratio data can provide additional information on the crustal structure, particularly a shallow crust with thick sediments, which is a good complement to the dispersion data. Joint inversion can also improve the resolution and reliability of the final model, which has been confirmed in traditional seismic inversion and in the 2-D joint inversion in our synthetic example.

\section{Conclusions}

Here, we provide an approach to calculating 2-D sensitivity kernels of the Rayleigh wave $\mathrm{ZH}$ ratio by using the adjoint-wavefield method. The results were also corroborated by the perturbation method and are consistent with previous results using 1-D sensitivity kernels. The 2-D sensitivity kernel of the $\mathrm{ZH}$ ratio has its preferred orientation toward the source. This explains why traditional $\mathrm{ZH}$ ratio measurements need good azimuthal coverage of the sources. We also found that the maximum sensitivity depth of the $\mathrm{ZH}$ ratio is similar to that of the phase velocity, which is deeper than the previous view from 1-D kernels. From synthetic examples with an undulated interface or a velocity anomaly, we found that the influences on the kernels of 2-D structures are significant. From these two inversion examples, we showed how the new 2-D kernels can be used to resolve lateral heterogeneity. When the $\mathrm{ZH}$ ratio data were jointly inverted with phases, the inversion result showed better resolution and less smearing. Yet measuring the $\mathrm{ZH}$ ratios from a single source-station pair is still challenging for ambient noise cross-correlations or earthquakes, and this is currently the main hindrance to putting the new 2-D kernels into practice.

\section{Acknowledgments}

We appreciate the constructive comments from two reviewers. This study was funded by the National Key R\&D Program of China (2016YFC0600301, 2018YFC1503400), the National Natural Science Foundation of China (41790464), Natural Science Foundation of Jiangsu Province of China (BK20190499), and the Fundamental Research Funds for the Central Universities (2019B0071428). The open source spectral-element software package SPECFEM2D used for this article is freely available via the Computational Infrastructure for Geodynamics (CIG; https://geodynamics.org/cig/software/specfem2d/).

\section{References}

Allmark, C., Curtis, A., Galetti, E., and de Ridder, S. (2018). Seismic attenuation from ambient noise across the north sea Ekofisk permanent array. J. Geophys. Res. Solid Earth, 123(10), 8691-8710. https://doi.org/10.1029/2017jb015419

Bao, X. Y., and Shen, Y. (2018). Full-waveform sensitivity kernels of componentdifferential traveltimes and $\mathrm{ZH}$ amplitude ratios for velocity and density tomography. J. Geophys. Res. Solid Earth, 123(6), 4829-4840. https://doi.org/10.1029/2017jb015421

Berbellini, A., Morelli, A., and Ferreira, A. M. G. (2017). Crustal structure of northern Italy from the ellipticity of Rayleigh waves. Phys. Earth Planet. Interiors, 265, 1-14. https://doi.org/10.1016/j.pepi.2016.12.005

Boore, D. M., and Toksöz, M. N. (1969). Rayleigh wave particle motion and crustal structure. Bull. Seismol. Soc. Am., 59(1), 331-346.

Brocher, T. M. (2005). Empirical relations between elastic wavespeeds and density in the Earth's crust. Bull. Seismol. Soc. Am., 95(6), 2081-2092. https://doi.org/10.1785/0120050077

Chen, P., Jordan, T. H., and Zhao, L. (2007). Full three-dimensional tomography: a comparison between the scattering-integral and adjoint-wavefield 
methods. Geophys. J. Int., 170(1), 175-181. https://doi.org/10.1111/j.1365246x.2007.03429.x

Chong, J. J., Ni, S. D., and Zhao, L. (2015). Joint inversion of crustal structure with the Rayleigh wave phase velocity dispersion and the $\mathrm{ZH}$ ratio. Pure Appl. Geophys., 172(10), 2585-2600. https://doi.org/10.1007/s00024-014-0902-z

Chong, J. J., Ni, S. D., Chu, R. S., and Somerville, P. (2016). Joint inversion of body-wave receiver function and Rayleigh-wave ellipticity. Bull. Seismol. Soc. Am., 106(2), 537-551. https://doi.org/10.1785/0120150075

Dahlen, F. A., and Baig, A. M. (2002). Fréchet kernels for body-wave amplitudes. Geophys. J. Int., 150(2), 440-466. https://doi.org/10.1046/j.1365246x.2002.01718.x

Ferreira, A. M., and Woodhouse, J. H. (2007a). Observations of long period Rayleigh wave ellipticity. Geophys. J. Int., 169(1), 161-169. https://doi.org/10.1111/j.1365-246x.2006.03276.x

Ferreira, A. M., and Woodhouse, J. H. (2007b). Source, path and receiver effects on seismic surface waves. Geophys. J. Int., 168(1), 109-132. https://doi.org/10.1111/j.1365-246x.2006.03092.x

Forbriger, T., Groos, L., and Schäfer, M. (2014). Line-source simulation for shallow-seismic data - Part 1: Theoretical background. Geophys. J. Int., 198(3), 1387-1404. https://doi.org/10.1093/gji/ggu199

Komatitsch, D., and Tromp, J. (2002a). Spectral-element simulations of global seismic wave propagation — I. Validation. Geophys. J. Int., 149(2), 390-412. https://doi.org/10.1046/j.1365-246x.2002.01653.x

Komatitsch, D., and Tromp, J. (2002b). Spectral-element simulations of global seismic wave propagation - II. Three-dimensional models, oceans, rotation and self-gravitation. Geophys. J. Int., 150(1), 303-318. https://doi.org/10.1046/j.1365-246x.2002.01716.x

Lin, F. C., Schmandt, B., and Tsai, V. C. (2012). Joint inversion of Rayleigh wave phase velocity and ellipticity using USArray: Constraining velocity and density structure in the upper crust. Geophys. Res. Lett., 39(12), L12303. https://doi.org/10.1029/2012gl052196

Lin, F. C., Tsai, V. C., and Schmandt, B. (2014). 3-D crustal structure of the western United States: application of Rayleigh-wave ellipticity extracted from noise cross-correlations. Geophys. J. Int., 198(2), 656-670. https://doi.org/10.1093/gji/ggu160

Liu, Q. Y., and Tromp, J. (2006). Finite-frequency kernels based on adjoint methods. Bull. Seismol. Soc. Am., 96(6), 2383-2397. https://doi.org/10.1785/0120060041

Liu, Q. Y., and Gu, Y. J. (2012). Seismic imaging: from classical to adjoint tomography. Tectonophysics, 566-567, 31-66. https://doi.org/10.1016/j.tecto.2012.07.006

Maupin, V. (2017). 3-D sensitivity kernels of the Rayleigh wave ellipticity. Geophys. J. Int., 211(1), 107-119. https://doi.org/10.1093/gji/ggx294

Shapiro, N. M., and Ritzwoller, M. H. (2002). Monte-Carlo inversion for a global shear-velocity model of the crust and upper mantle. Geophys. J. Int., 151(1), 88-105. https://doi.org/10.1046/j.1365-246x.2002.01742.x

Shen, Y., Zhang, Z. G., and Zhao, L. (2008). Component-dependent Fréchet sensitivity kernels and utility of three-component seismic records. Bull. Seismol. Soc. Am., 98(5), 2517-2525. https://doi.org/10.1785/0120070283

Tanimoto, T., and Alvizuri, C. (2006). Inversion of the HZ ratio of microseisms for S-wave velocity in the crust. Geophys. J. Int., 165(1), 323-335. https://doi.org/10.1111/j.1365-246x.2006.02905.x

Tanimoto, T., and Rivera, L. (2008). The ZH ratio method for long-period seismic data: sensitivity kernels and observational techniques. Geophys. J. Int., 172(1), 187-198. https://doi.org/10.1111/j.1365-246x.2007.03609.x

Tanimoto, T., and Tsuboi, S. (2009). Variational principle for Rayleigh wave ellipticity. Geophys. J. Int., 179(3), 1658-1668. https://doi.org/10.1111/j.1365246x.2009.04360.x

Tanimoto, T., Yano, T., and Hakamata, T. (2013). An approach to improve Rayleigh-wave ellipticity estimates from seismic noise: application to the Los Angeles Basin. Geophys. J. Int., 193(1), 407-420. https://doi.org/10.1093/gji/ggs123

Tape, C., Liu, Q. Y., and Tromp, J. (2007). Finite-frequency tomography using adjoint methods-Methodology and examples using membrane surface waves. Geophys. J. Int., 168(3), 1105-1129. https://doi.org/10.1111/j.1365246x.2006.03191.x

Tromp, J., Tape, C., and Liu, Q. Y. (2005). Seismic tomography, adjoint methods, time reversal and banana-doughnut kernels. Geophys. J. Int., 160(1), 195-216. https://doi.org/10.1111/j.1365-246x.2004.02453.x

Workman, E., Lin, F. C., and Koper, K. D. (2016). Determination of Rayleigh wave ellipticity across the Earthscope Transportable Array using single-station and array-based processing of ambient seismic noise. Geophys. J. Int., 208(1), 234-245. https://doi.org/10.1093/gji/ggw381

Yang, Y., Yao, H. J., Wu, H. X., Zhang, P., and Wang, M. M. (2020). A new crustal shear-velocity model in Southwest China from joint seismological inversion and its implications for regional crustal dynamics. Geophys. J. Int., 220(2), 1379-1393. https://doi.org/10.1093/gji/ggz514

Yano, T., Tanimoto, T., and Rivera, L. (2009). The ZH ratio method for longperiod seismic data: inversion for S-wave velocity structure. Geophys. J. Int., 179(1), 413-424. https://doi.org/10.1111/j.1365-246x.2009.04293.x

Yuan, Y., Yao, H. J., and Qin Y. (2016). Joint inversion of Rayleigh wave verticalhorizontal amplitude ratios and dispersion based on the Neighborhood Algorithm and its application. Chinese J. Geophys. (in Chinese), 59(3), 959-971. https://doi.org/10.6038/cjg20160318

Yuan, Y. O., Simons, F. J., and Tromp, J. (2016). Double-difference adjoint seismic tomography. Geophys. J. Int., 206(3), 1599-1618. https://doi.org/10.1093/gji/ggw233

Zhang, C., Yao, H. J., Liu, Q. Y., Zhang, P., Yuan, Y. O., Feng, J. K., and Fang, L. H. (2018). Linear array ambient noise adjoint tomography reveals intense crust-mantle interactions in North China craton. J. Geophys. Res. Solid Earth, 123(1), 368-383. https://doi.org/10.1002/2017jb015019

Zhao, L., Jordan, T. H., Olsen, K. B., and Chen, P. (2005). Fréchet kernels for imaging regional Earth structure based on three-dimensional reference models. Bull. Seismol. Soc. Am., 95(6), 2066-2080. https://doi.org/10.1785/0120050081

Zhang, Z. G., and Shen, Y. (2008). Cross-dependence of finite-frequency compressional waveforms to shear seismic wave speeds. Geophys. J. Int., 174(3), 941-948. https://doi.org/10.1111/j.1365-246x.2008.03840.x

Zhou, Y., Dahlen, F. A., and Nolet, G. (2004). Three-dimensional sensitivity kernels for surface wave observables. Geophys. J. Int., 158(1), 142-168. https://doi.org/10.1111/j.1365-246x.2004.02324.x 